\title{
Super-Weyl Invariant 2D Supergravity, Anomaly and WZ Action
}

\author{
K.KAMIMURA $^{\dagger}$ AND R.KURIKI $^{\dagger \dagger}$ \\ $\dagger^{\dagger}$ Department of Physics, Toho University \\ Funabashi, 274 JAPAN \\ ${ }^{\dagger \dagger}$ Ochanomizu University, Department of Physics, Faculty of Science \\ Otsuka-Machi, Bunkyo-Ku Tokyo, 112 JAPAN \\ kamimura@ph.sci.toho-u.ac.jp, r-kuriki@fs.cc.ocha.ac.jp
}

\begin{abstract}
We present a candidate of anomaly and Wess Zumino action of the two dimensional supergravity coupling with matters in a super-Weyl invariant regularization. It is a generalization of the Weyl and the area preserving diffeomorphism invariant formulation of two dimensional gravity theory.
\end{abstract}

July 1997

TOHO-FP-9755

OCHA-PP-102 


\section{Introduction}

Recently two dimensional gravity theory coupling with scalar matter is discussed in the Weyl invariant regularization [1] [2] [3] [4] [5] [n]. In the standard diffeomorphism invariant formulation the Weyl transformations become anomalous and the gauge parameter of Weyl symmetry is propagating at the quantum level [7]. In contrast to the regularization which respects the two dimensional diffeomorphisms, the Weyl invariant formulation causes a breaking of the two dimensional diffeomorphisms. However the two dimensional diffeomorphisms ( Diff $_{2}$ ) is not fully broken but the area preserving diffeomorphism (SDiff) still remains invariant. The form of anomaly which possesses the Weyl and the area-preserving diffeomorphism invariances has been constructed. Moreover a local form of the Wess-Zumino (WZ) term is obtained using the general formalism in terms of the coset coordinate of $\frac{\text { Diff } 2}{\text { SDiff }}$ [6].

In this paper we will extend the discussions on the Weyl invariant gravity theory to two dimensional (2D) supergravity theory. We present a candidate of anomaly satisfying the Wess Zumino consistency condition assuming super-Weyl invariant reguralizations. We also obtain the local WZ action for the anomaly. Although the diffeomorphism symmetries become anomalous the SDiff remains unbroken as in the bosonic case. In [8] the author investigated an extension of area-preserving structure to 2D supergravity and conjectured a non-local form of effective action invariant under the super extension of area-preserving diffeomorphism . However the super-Weyl invariant anomaly which we will give in this paper does not allow such super extension of the SDiff. The two dimensional local supersymmetries are fully anomalous in the present super Weyl invariant formulation.

This paper is organized as follows. In section 2 we give a brief review of the $2 \mathrm{D}$ supergravity invariant under diffeomorphism and the two dimensional supersymmetry. The super-Weyl anomaly and WZ action are also given there. In section 3 we introduce some super-Weyl invariant variables using a canonical transformation. The super-Weyl invariant anomaly and WZ action is given in section 4. We present some discussions in the last section

\section{Super-Weyl anomaly and WZ term}

Before going into the Super-Weyl invariant formulation of the two dimensional supergravity we briefly review the diffeomorphism invariant formulation since our main discussions are based on them. We follow the discussion of the reference [9] and use the notations.

The two dimensional supergravity theory can be formulated by the Neveu-SchwarzRamond superstring model. The classical action is given by

$$
S_{0}=-\int d^{2} x e\left[\frac{1}{2}\left(g^{\alpha \beta} \partial_{\alpha} X \partial_{\beta} X-i \bar{\psi} \rho^{\alpha} \nabla_{\alpha} \psi\right)+\bar{\chi}_{\alpha} \rho^{\beta} \rho^{\alpha} \psi \partial_{\beta} X+\frac{1}{4}(\bar{\psi} \psi)\left(\bar{\chi}_{\alpha} \rho^{\beta} \rho^{\alpha} \chi_{\beta}\right)\right],
$$

where $X^{\mu}, \psi^{\mu}, e_{\alpha}{ }^{a}$ and $\chi_{\alpha}$ are respectively the bosonic and fermionic string coordinates, the zwei-bein and gravitino fields. The space-time indices is $\mu=0, \ldots, D-1$, which is 
abbreviated in (2.1), and the world-sheet indices is $\alpha=0,1$. The covariant derivative is defined by

$$
\nabla_{\alpha} \psi \equiv\left(\partial_{\alpha}+\frac{1}{2} \varpi_{\alpha} \rho_{5}\right) \psi, \quad \varpi_{\alpha}=\frac{1}{e} e_{\alpha}{ }^{a} \epsilon^{\beta \gamma} \partial_{\beta} e_{\gamma a}+2 i \bar{\chi}_{\alpha} \rho_{5} \rho^{\beta} \chi_{\beta} .
$$

The action has local symmetries under the two dimensional diffeomorphisms, the local Lorentz, the two dimensional world-sheet local supersymmetries, the Weyl rescaling, the local fermionic transformations. The last one is a result from the property of the two dimensional gamma matrixes. We will refer the Weyl rescaling and the local fermionic transformations as 'super-Weyl transformations (SW)'.

In the regularization which respects Diff 2 and the two dimensional world-sheet local supersymmetries the SW symmetries cannot be maintained but become anomalous. The SW anomaly satisfying the WZ consistency condition $\delta \mathcal{A}=0$, has been given by

$$
\begin{aligned}
\mathcal{A}= & -i \hbar k \int d^{2} x\left[R C_{W}-4 \bar{T} \rho_{5} \eta_{W}\right], \\
& R \equiv 2\left(\epsilon^{\alpha \beta} \partial_{\alpha} \varpi_{\beta}\right), \quad T \equiv \epsilon^{\alpha \beta} \nabla_{\alpha} \chi_{\beta} .
\end{aligned}
$$

where $k$ is a constant and $C_{W}$ and $\eta_{W}$ are ghost variables corresponding to the Weyl and the local fermionic transformations respectively.

It is well-known that we can construct a local counter term for the anomaly by a introducing extra field, the super Liouville mode. The following general method [10 the WZ action is constructed [9] using Wely degrees of freedom $\sigma$ and super-Wely degrees of freedom $\eta$ as,

$$
\mathcal{M}_{1}=\hbar k S^{S L}=\hbar k\left(S_{0}^{S L}+S_{1}^{S L}\right)
$$

with

$$
\begin{aligned}
S_{0}^{S L} & =-\int d^{2} x e\left\{\frac{1}{2}\left(g^{\alpha \beta} \partial_{\alpha} \sigma \partial_{\beta} \sigma-i \bar{\eta} \rho^{\alpha} \nabla_{\alpha} \eta\right)-\bar{\chi}_{\alpha} \rho^{\beta} \rho^{\alpha} \eta \partial_{\beta} \sigma+\frac{1}{4} \bar{\eta} \eta \bar{\chi}{ }_{\alpha} \rho^{\beta} \rho^{\alpha} \chi_{\beta}\right\} \\
S_{1}^{S L} & =-\int d^{2} x\left[R \sigma-4 \bar{T} \rho_{5} \eta\right] .
\end{aligned}
$$

The WZ action verifies $\delta \mathcal{M}_{1}=i \mathcal{A}$ off shell and the BRST transformations of the extra variables are given by

$$
\begin{aligned}
& \delta_{0} \sigma=\partial_{\alpha} \sigma C^{\alpha}-\bar{\eta} \omega-C_{W} \\
& \delta_{0} \eta=\partial_{\alpha} \eta C^{\alpha}+\frac{1}{2} \rho_{5} \eta C_{L}+i \rho^{\alpha} \omega\left(\partial_{\alpha} \sigma+\bar{\eta} \chi_{\alpha}\right)-\frac{1}{4} \eta C_{W}-\eta_{W}
\end{aligned}
$$

where the ghosts for the diffeomorphisms, the local Lorentz and the two dimensional world-sheet local supersymmetries are denoted as $C_{\alpha}, C_{L}$ and $\omega$ respectively.

The field-antifield formalism is one of the most useful formalism to investigate the gauge structure. The classical master equation (CME), $(S, S)=0$, which is defined in the local functional space of fields and antifields, encodes all gauge structure of the theory 
[11]. The minimal proper solution of the CME is

$$
\begin{aligned}
S & =S_{0} \\
& +X^{*}\left(\partial_{\alpha} X C^{\alpha}+\bar{\psi} \omega\right) \\
& +\psi^{*}\left(\partial_{\alpha} \psi C^{\alpha}+\frac{1}{2} \rho_{5} \psi C_{L}-\frac{1}{4} \psi C_{W}-i \rho^{\alpha} \omega\left(\partial_{\alpha} X-\bar{\psi} \chi_{\alpha}\right)\right) \\
& +e^{\alpha *}\left(\partial_{\beta} e_{\alpha}{ }^{a} C^{\beta}+e_{\beta}{ }^{a} \partial_{\alpha} C^{\beta}+\epsilon_{b}^{a} e_{\alpha}{ }^{b} C_{L}+\frac{1}{2} e_{\alpha}{ }^{a} C_{W}+2 i \overline{\chi_{\alpha}} \rho^{a} \omega\right) \\
& +\chi^{\alpha *}\left(\partial_{\beta} \chi_{\alpha} C^{\beta}+\chi_{\beta} \partial_{\alpha} C^{\beta}+\frac{1}{2} \rho_{5} \chi_{\alpha} C_{L}+\frac{1}{4} \chi_{\alpha} C_{W}+\frac{i}{4} \rho_{\alpha} \eta_{W}+\nabla_{\alpha} \omega\right) \\
& +C_{\alpha}{ }^{*}\left(\partial_{\beta} C^{\alpha} C^{\beta}-i \bar{\omega} \rho^{\alpha} \omega\right) \\
& +C_{L}{ }^{*}\left(\partial_{\alpha} C_{L} C^{\alpha}+\frac{1}{2} \bar{\omega} \rho_{5} \eta_{W}-\varpi_{\alpha} i \bar{\omega} \rho^{\alpha} \omega\right) \\
& +\omega^{*}\left(\partial_{\alpha} \omega C^{\alpha}+\frac{1}{2} \rho_{5} \omega C_{L}+\frac{1}{4} \omega C_{W}+\chi_{\alpha}\left(i \bar{\omega} \rho^{\alpha} \omega\right)\right) \\
& +C_{W}{ }^{*}\left(\partial_{\alpha} C_{W} C^{\alpha}-\bar{\omega} \eta_{W}\right) \\
& +\eta_{W}{ }^{*}\left(\partial_{\alpha} \eta_{W} C^{\alpha}+\frac{1}{2} \rho_{5} \eta_{W} C_{L}-\frac{1}{4} \eta_{W} C_{W}-i \rho^{\alpha} \omega\left(\partial_{\alpha} C_{W}+\bar{\chi}_{\alpha} \eta_{W}\right)-\frac{4}{e} \omega\left(\bar{\omega} \rho_{5} T\right)\right) \\
& -\frac{1}{2 e}\left(\psi^{*} \omega\right)\left(\psi^{*} \omega\right) .
\end{aligned}
$$

It is easy to read the BRST transformations of the fields. It contains a quadratic term of the anti-field $\psi^{*}$ reflecting the fact that the two dimensional local supersymmetry algebra on the spinor $\psi$ closes only on shell of its equation of motion.

\section{Super-Weyl invariant variables}

Here we will consider the SW invariant formulation. In order to maintain the manifest SW invariance it is useful to introduce the SW invariant variables [8]

$$
\begin{aligned}
\tilde{X} & =X, & \tilde{\psi} & =e^{1 / 4} \psi, \\
\tilde{e}_{\alpha}^{a} & =\frac{1}{e^{1 / 2}} e_{\alpha}^{a}, & \tilde{\chi}_{\alpha} & =\frac{-1}{2 e^{1 / 4}} \rho^{\beta} \rho_{\alpha} \chi_{\beta} .
\end{aligned}
$$

They are not independent but satisfy identically

$$
\operatorname{det} \tilde{e}_{\alpha}^{a}=1, \quad \tilde{\rho}^{\alpha} \tilde{\chi}_{\alpha}=0 \text {, }
$$

where Dirac matrix $\tilde{\rho}^{\alpha}(x)$ is defined using $\tilde{e}_{\alpha}^{a}$ and the indices of $\tilde{\chi}^{\alpha}$ and $\tilde{\rho}^{\alpha}$ 's are lifted and lowered by SW invariant metric $\tilde{g}^{\alpha \beta}$ and $\tilde{g}_{\alpha \beta}$ defined by $\tilde{e}_{\alpha}{ }^{b}$ and $\tilde{e}^{\beta}{ }_{a}$.

Since the classical action $S_{0}$ in $(2.1)$ is invariant under SW transformations it is expressed in terms of the invariant variables

$$
\tilde{S}_{0}=-\int d^{2} x\left[\frac{1}{2}\left(\tilde{g}^{\alpha \beta} \partial_{\alpha} \tilde{X} \partial_{\beta} \tilde{X}-i \overline{\tilde{\psi}} \tilde{\rho}^{\alpha} \tilde{\nabla}_{\alpha} \tilde{\psi}\right)-2 \overline{\tilde{\chi}}^{\beta} \tilde{\psi} \partial_{\beta} \tilde{X}-\frac{1}{2}(\overline{\tilde{\psi}} \tilde{\psi})\left(\overline{\tilde{\chi}_{\beta}} \tilde{\chi}_{\beta}\right)\right]
$$


where the covariant derivative, which is invariant under SW transformations, is

$$
\tilde{\nabla}_{\alpha} \tilde{\psi}=\left(\partial_{\alpha}+\frac{1}{2} \rho_{5} \tilde{\varpi}_{\alpha}\right) \tilde{\psi}, \quad \tilde{\varpi}_{\alpha}=\tilde{e}_{\alpha}^{a} \epsilon^{\rho \sigma} \partial_{\rho} \tilde{e}_{\sigma a}
$$

The BRST transformations of new variables are obtained from (2.9) by making a canonical transformation in the sense of anti-bracket formalism. The transformation (3.1) is generated by the generating function

$$
\begin{aligned}
W\left(\phi, \tilde{\phi}^{*}\right) & =\tilde{X}^{*} X+\tilde{\psi}^{*}\left(e^{\frac{1}{4}} \psi\right)+\tilde{e}_{a}^{* \alpha}\left(\frac{e_{\alpha}^{a}}{e^{\frac{1}{2}}}\right)+E^{*} e \\
& +\tilde{\chi}^{* \alpha}\left(\frac{-1}{2 e^{\frac{1}{4}}} \rho^{\beta} \rho_{\alpha} \chi_{\beta}\right)+\hat{\chi}^{*}\left(i e^{\frac{1}{4}} \rho^{\alpha} \chi_{\alpha}\right) \\
& +\tilde{C}_{L}^{*}\left(C_{L}-i \bar{\omega} \rho_{5} \rho^{\alpha} \chi_{\alpha}\right)+\tilde{C}_{W}^{*}\left(C_{W}+2 i \bar{\omega} \rho^{\alpha} \chi_{\alpha}\right) \\
& +\tilde{C}_{\alpha}^{*} C^{\alpha}+\tilde{\omega}^{*}\left(\frac{\omega}{e^{\frac{1}{4}}}\right)+\tilde{\eta}_{W}^{*}\left(e^{\frac{1}{4}} \eta_{W}\right) .
\end{aligned}
$$

It also defines SW invariant ghosts $\tilde{C}^{\alpha}, \tilde{\omega}$ and $\tilde{C}_{L}$ and SW non-invariant fields $E, \hat{\chi}, \tilde{C}_{W}$ and $\tilde{\eta}_{W}$. In terms of new variables the solution $S$ of CME in (2.9) becomes a sum of two terms

$$
\tilde{S}=\tilde{S}_{i n v}+\tilde{S}_{n o n}
$$

The first term $\tilde{S}_{i n v}$ contains only SW invariant variables as

$$
\begin{aligned}
\tilde{S}_{i n v}= & \tilde{S}_{0}+ \\
& +\tilde{X}^{*}\left(\partial_{\alpha} \tilde{X} C^{\alpha}+\overline{\tilde{\psi}} \tilde{\omega}\right) \\
& +\tilde{\psi}^{*}\left(\partial_{\alpha} \tilde{\psi} C^{\alpha}+\frac{1}{2} \rho_{5} \tilde{\psi} \tilde{C}_{L}+\frac{1}{4} \tilde{\psi}\left(\partial_{\beta} C^{\beta}\right)-i \tilde{\rho}^{\alpha} \tilde{\omega}\left(\partial_{\alpha} \tilde{X}-\overline{\tilde{\psi}} \tilde{\chi}_{\alpha}\right)\right) \\
& +\tilde{e}_{a}^{\alpha *}\left(\partial_{\beta} \tilde{e}_{\alpha}{ }^{a} C^{\beta}+\tilde{e}_{\beta}{ }^{a} \partial_{\alpha} C^{\beta}-\frac{1}{2} \tilde{e}_{\alpha}{ }^{a}\left(\partial_{\beta} C^{\beta}\right)+\epsilon_{b}^{a} \tilde{e}_{\alpha}{ }^{b} \tilde{C}_{L}+2 i \overline{\tilde{\chi}_{\alpha}} \rho^{a} \tilde{\omega}\right) \\
& +\tilde{\chi}^{\alpha *}\left(\partial_{\beta} \tilde{\chi}_{\alpha} C^{\beta}+\tilde{\chi}_{\beta} \partial_{\alpha} C^{\beta}-\frac{1}{4} \tilde{\chi}_{\alpha}\left(\partial_{\beta} C^{\beta}\right)+\frac{1}{2} \rho_{5} \tilde{\chi}_{\alpha} \tilde{C}_{L}+\tilde{\nabla}_{\alpha} \tilde{\omega}-\frac{i}{2} \tilde{\rho}_{\alpha} \Omega\right) \\
& +C_{\alpha}^{*}\left(\partial_{\beta} C^{\alpha} C^{\beta}-i \overline{\tilde{\omega}} \tilde{\rho}^{\alpha} \tilde{\omega}\right) \\
& +\tilde{C}_{L}^{*}\left(\partial_{\alpha} \tilde{C}_{L} C^{\alpha}-\overline{\tilde{\omega}} \rho_{5} \Omega-\tilde{\omega}_{\alpha} i \overline{\tilde{\omega}} \tilde{\rho}^{\alpha} \tilde{\omega}\right) \\
& +\tilde{\omega}^{*}\left(\partial_{\alpha} \tilde{\omega} C^{\alpha}+\frac{1}{2} \rho_{5} \tilde{\omega} \tilde{C}_{L}-\frac{1}{4} \tilde{\omega}\left(\partial_{\beta} C^{\beta}\right)+\tilde{\chi}_{\alpha}\left(i \overline{\tilde{\omega}} \tilde{\rho}^{\alpha} \tilde{\omega}\right)\right) \\
& -\frac{1}{2}\left(\tilde{\psi}^{*} \tilde{\omega}\right)\left(\tilde{\psi}^{*} \tilde{\omega}\right) .
\end{aligned}
$$

where

$$
\Omega \equiv i \tilde{\rho}^{\alpha} \tilde{\nabla}_{\alpha} \tilde{\omega}+2\left(\overline{\tilde{\chi}^{\gamma}} \tilde{\chi}_{\gamma}\right) \tilde{\omega} \text {. }
$$

The remaining terms in $\tilde{S}_{n o n}$ contain the SW non invariant variables. Since the transformation is canonical, preserving the anti-brackets, $\tilde{S}$ is still satisfying the CME, $(\tilde{S}, \tilde{S})=0$. Furthermore the first term $\tilde{S}_{i n v}$ satisfies CME $\left(\tilde{S}_{i n v}, \tilde{S}_{i n v}\right)=0$ by itself. In other words we have introduced new ghosts $\tilde{C}_{L}, \tilde{C}_{W}$, etc. so that their BRST transformations are closed. Actually if we start from the action $\tilde{S}_{0}$ in (3.3) we obtain the $\tilde{S}_{i n v}$ as the solution of CME. 


\section{SW invariant Anomaly and WZ action}

The SW invariant anomaly is described by the SW invariant variables. In order to find it, we first recognize that the forms of the BRST transformations of new variables have the same structures as the corresponding original variables. $\tilde{S}_{i n v}$ in (3.7) is obtained from $S$ in (2.9) by the following replacements :

$$
e_{\alpha}^{a} \rightarrow \tilde{e}_{\alpha}^{a}, \quad \chi_{\alpha} \rightarrow \tilde{\chi}_{\alpha}, \quad \omega \rightarrow \tilde{\omega}, \quad C_{L} \rightarrow \tilde{C}_{L},
$$

and

$$
C_{W} \rightarrow-\left(\partial_{\alpha} C^{\alpha}\right), \quad \eta_{W} \rightarrow-2 \Omega .
$$

That is the Weyl ghost $C_{W}$ is replaced by a combination of diffeomorphism ghost $-(\partial C)$ and the super Weyl ghost $\eta_{W}$ is replaced by that of supersymmetry ghost $-2 \Omega$. Furthermore the transformation properties of $\left(\partial_{\alpha} C^{\alpha}\right)$ and $\Omega$ are

$$
\begin{aligned}
\delta(\partial C) & =\partial_{\beta}(\partial C) C^{\beta}-2 \overline{\tilde{\omega}} \Omega \\
\delta \Omega & =\partial_{\alpha} \Omega C^{\alpha}+\frac{1}{2} \rho_{5} \Omega \tilde{C}_{L}+\frac{1}{4} \Omega(\partial C)-\frac{i}{2} \tilde{\rho}^{\alpha} \tilde{\omega}\left(\partial_{\alpha}(\partial C)+2 \overline{\tilde{\chi}_{\alpha}} \Omega\right)+2 \tilde{\omega}\left(\overline{\tilde{T}} \rho_{5} \tilde{\omega}\right)
\end{aligned}
$$

which are also obtained from $\delta C_{W}$ and $\delta \eta_{W}$ by the same replacements.

Guided by the transformation properties of the new variables one can deduce a candidate of the SW invariant anomaly satisfying WZ consistency condition. Knowing the SW non-invariant anomaly (2.3) and making the replacements (4.1) and (4.2) we obtain

$$
\mathcal{A}^{S W}=-i \hbar k \int d^{2} x\left(-\tilde{R}(\partial C)+8 \tilde{T} \rho_{5} \Omega\right)
$$

where

$$
\tilde{R}=2 \epsilon^{\alpha \beta} \partial_{\alpha} \tilde{\varpi}_{\beta}, \quad \tilde{T}=\epsilon^{\alpha \beta} \tilde{\nabla}_{\alpha} \tilde{\chi}_{\beta} .
$$

It is straightforward to show that it satisfies the WZ consistency condition

$$
\delta \mathcal{A}^{S W}=0 \text {. }
$$

That is $\mathcal{A}^{S W}$ is the candidate of a SW invariant anomaly which would be obtained in the SW invariant regularizations at one loop level.

Next we find the WZ action $\mathcal{M}_{1}^{S W}$ for the SW invariant anomaly (4.5). It is the solution of

$$
\delta \mathcal{M}_{1}^{S W}(\phi, \theta)=i \mathcal{A}^{S W}
$$

and is found as a function of the classical fields $\phi$ and the extra variables $\theta$. The latter are essentially anomalous gauge degrees of freedoms whose dynamical action is the WZ term.

In the case of gravity they are finite diffeomorphism functions $f^{\mu}(x)$. Since the SDiff is non anomalous the WZ action is a function of only one degrees of freedom $\Theta$ parametrizing the coset $\frac{D i f f}{\text { SDiff }}$. In the present case the local supersymmetry is also anomalous. The WZ action is expected to be a function of a bosonic extra field $\Theta$ and a fermionic extra spinor $\Psi$. 
The explicit form of the WZ action is obtained from the form of the SW non invariant WZ term, i.e. super Liouville action (2.5)-(2.7), by the replacements (4.1) and

$$
\sigma \rightarrow-\Theta, \quad \eta \rightarrow-\Psi
$$

as

$$
\mathcal{M}_{1}^{S W}=\hbar k S^{S W}=\hbar k\left(S_{0}^{S W}+S_{1}^{S W}\right),
$$

with

$$
\begin{aligned}
S_{0}^{S W} & =-\int d^{2} x\left[\frac{\tilde{g}^{\alpha \beta}}{2} \partial_{\alpha} \Theta \partial_{\beta} \Theta-\frac{i}{2} \bar{\Psi} \tilde{\rho}^{\alpha} \partial_{\alpha} \Psi+2\left(\overline{\tilde{\chi}^{\beta}} \Psi\right) \partial_{\beta} \Theta-\frac{1}{2}(\bar{\Psi} \Psi)\left(\overline{\tilde{\chi}^{\alpha}} \tilde{\chi}_{\alpha}\right)\right] \\
S_{1}^{S W} & =-\int d^{2} x\left[-\tilde{R} \Theta+4 \overline{\tilde{T}} \rho_{5} \Psi\right]
\end{aligned}
$$

It is noted that $S_{0}^{S W}$ is obtained from the classical action $\tilde{S}_{0}$ in (3.3) by the replacements $\tilde{X} \rightarrow \Theta$ and $\tilde{\psi} \rightarrow-\Psi$ and $S_{1}^{S W}$ is obtained from the anomaly by the replacements $(\partial C) \rightarrow \Theta$ and $2 \Omega \rightarrow \Psi$. The WZ action verifies

$$
\delta \mathcal{M}_{1}^{S W}=i \mathcal{A}^{S W}
$$

off shell if the BRST transformations of the extra variables $\Theta$ and $\Psi$ are introduced as

$$
\begin{aligned}
& \delta \Theta=\partial_{\alpha} \Theta C^{\alpha}-\bar{\Psi} \omega-(\partial C) \\
& \delta \Psi=\partial_{\alpha} \Psi C^{\alpha}+\frac{1}{4} \Psi(\partial C)+\frac{1}{2} \rho_{5} \Psi \tilde{C}_{L}+i \tilde{\rho}^{\alpha} \omega\left(\partial_{\alpha} \Theta+\bar{\Psi} \tilde{\chi}_{\alpha}\right)-2 \Omega .
\end{aligned}
$$

They are deduced from the SW non invariant ones (2.8) by the replacements (4.1), (4.2) and (4.9).

\section{Discussions}

In this paper we have discussed two dimensional supergravity under a SW invariant regularization. We have found the candidate anomaly and the Wess Zumino action. They are deduced from the properties of the BRST transformations of the SW invariant supergravity multiplet. The crucial property is the correspondence of the BRST transformations between the original and the SW invariant variables. The latter is obtained from the former by the replacements (4.1) and (4.2),

$$
C_{W} \rightarrow-\left(\partial_{\alpha} C^{\alpha}\right), \quad \eta_{W} \rightarrow-2 \Omega=-2\left[i \tilde{\rho}^{\alpha} \tilde{\nabla}_{\alpha}+2\left(\overline{\tilde{\chi}^{\gamma}} \tilde{\chi}_{\gamma}\right)\right] \tilde{\omega} .
$$

In the anomaly (4.5) the diffeomorphism ghosts $C^{\alpha}$ appears in the combination of $(\partial C)$. It means that the diffeomorphism transformations with the infinitesimal parameter $\epsilon^{\alpha}$ satisfying

$$
\left(\partial_{\alpha} \epsilon^{\alpha}\right)=0, \quad \text { i.e. } \quad \epsilon^{\alpha}=\epsilon^{\alpha \beta} \partial_{\beta} \epsilon,
$$

are not anomalous. The area preserving diffeomorphisms remain non anomalous as in the bosonic theory. On the other hand supersymmetry ghosts $\omega$ appears in the combination 
of $\Omega$. The local supersymmetry transformations would be non anomalous if the spinor parameter $\tilde{\varepsilon}$ is satisfying

$$
\left[i \tilde{\rho}^{\alpha} \tilde{\nabla}_{\alpha}+2\left(\overline{\tilde{\chi}^{\gamma}} \tilde{\chi}_{\gamma}\right)\right] \tilde{\varepsilon}=0 .
$$

However there found no local solutions of (5.3) unlike the case of (5.2). Furthermore the WZ action (4.10) has no additional gauge symmetry. In the last paper [6] we have shown that there appears additional gauge invariance of the WZ action if we would have introduced extra variables associated to the non anomalous transformations. It follows the degree of freedom of $\Psi$ agrees with that of the fermionic anomalous transformations. We can conclude that the all two dimensional world-sheet supersymmetries become anomalous under the SW invariant regularization. It is also consistent with the fact that the number of independent anomalies should be unchanged under different regularizations. It is equal in the SW non-invariant and SW invariant regularizations.

The anomaly (4.5) would be obtained by the Pauli-Villars regularization using the counter term

$$
\tilde{S}^{M}=-\int d^{2} x \frac{1}{2}\left[M^{2} \tilde{X} \tilde{X}-i M \overline{\tilde{\psi}} \tilde{\psi}\right]
$$

It naturally breaks the local supersymmetries and area non-preserving diffeomorphism while it is invariant under area preserving diffeomorphism, local Lorentz and SW transformations.

The BRST transformation of the extra variables (4.15) is nilpotent only on shell of equation of WZ action, $\left[S^{S W}\right]_{\Psi}=0$,

$$
\delta^{2} \Theta=0, \quad \delta^{2} \Psi=\tilde{\omega}\left(\overline{\tilde{\omega}}\left[S^{S W}\right]_{\Psi}\right) \sim 0 .
$$

The similar situation happened in the case of SW non-invariant formalism [9]. As in the latter case it requires an additional term

$$
-\frac{1}{2}\left(\Psi^{*} \tilde{\omega}\right)\left(\Psi^{*} \tilde{\omega}\right)
$$

in the quantum action. However the reasons of off shell non nilpotency are quite different. In the latter the off shell non nilpotency arises from the fact that the non-anomalous transformations ( local susy ) do not close but give anomalous ( super-Weyl ) transformations. In the present case the non anomalous transformations close by themselves. The off shell non nilpotency comes from the fact that the local supersymmetry transformations for the spinor $\Psi$ close only on shell of its equation of motions.

It is interesting to find the relation of the extra variables $\Theta$ and $\Psi$ with the local gauge degrees of freedoms. In the case of two dimensional gravity theory $\Theta$ is expressed by the finite diffeomorphism function $f^{\alpha}(x)$ as

$$
\Theta(x)=\ln \left(\Delta^{f}\right)_{x=F(x)}, \quad \text { with } \quad F(f(x))=x .
$$

The general framework to obtain such relations has been established [6]. It is also shown

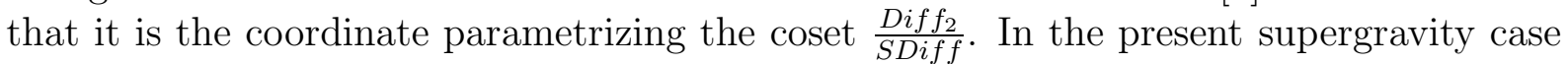
the extra variables $\Theta$ and $\Psi$ are regarded as the coordinates parametrizing the coset 
$\frac{D i f f_{2} \times S U S Y_{\text {local }}}{\text { SDiff }}$. The analysis in the general formalism and the explicit form of the extra variables in terms of finite gauge transformation parameters will be discussed elsewhere.

\section{Acknowledgments}

The authors would like to thank Prof.J.Gomis and Prof.D.R.Karakhanyan for helpful discussions.

\section{References}

[1] D. Karakhanyan, R.P.Manvelyan, and R.L.Mkrtchyan, Phys. Lett. B329, 185 (1994).

[2] R. JACKIw, Another view on Massless Matter-Gravity Fields in Two Dimensions, hep-th/9601016.

[3] G.Amelino-Camelia, A.Bak and D.Seninara, Phys. Lett. B354, 213 (1995).

[4] J.G.Zhou,Y.G.Miao,J.Q.Liang,H.J.W.Mueller-Kirsten,Z.Zhang Z.Phy. C73, 563 (1997)

[5] J.Cruz, J.Navarro-Salas Phys.Lett. B375, 47 (1996)

[6] J.Gomis, K. Kamimura, and R. Kuriki, Nucl. Phys. B496 465 (1997).

[7] A. Polyakov, Mod. Phys. Lett A2,893 (1987)

[8] D.R.Karakhanyan, Phys.Lett. B365, 56 (1996)

[9] J.Gomis, K. Kamimura, and R. Kuriki, Nucl. Phys. B471 513 (1996).

[10] J.Gomis And J.Paris Nucl. Phys. B395, 288 (1993), Nucl. Phys. B431, 378 (1994).

J. Gomis, J. M. Pons And F. Zamora, Nucl. Phys. B446, 223 (1995),

J. Gomis, K. Kamimura, J. M. Pons and F. Zamora, Nucl. Phys. B458, 387 (1996).

[11] M.Henneaux And C.Teitelboim, Quantization of Gauge Systems ( Princeton Univ. Press, Princeton 1992 )

0 W.Troost and A.Van Proeyen, An introduction to Batalin-Vilkovisky lagrangian quantization, Leuven Notes in Math. Theor. Phys. in preparation. J.Gomis, J.París And S.SAmuel, Phys. Rep. 259 (1995) 1. 